\title{
Differentiating combined pulmonary fibrosis and emphysema from pure emphysema: utility of late gadolinium- enhanced MRI
}

Hannah Fleming ${ }^{1}$, Simon M. Clifford ${ }^{1}$, Aoife Haughey ${ }^{1}$, Roisin MacDermott ${ }^{1}$, Niall McVeigh ${ }^{1,2}$, Gerard M. Healy ${ }^{1}$, Lisa Lavelle', Suhny Abbara ${ }^{3}$, David J. Murphy', Aurelie Fabre ${ }^{2,4}$, Edward McKone ${ }^{2,5}$, Cormac McCarthy ${ }^{2,5}$, Marcus Butler ${ }^{2,5}$, Peter Doran ${ }^{6}$, David A. Lynch ${ }^{7}$, Michael P. Keane ${ }^{2,5}$ and Jonathan D. Dodd ${ }^{1,2^{*}}$ (D)

\begin{abstract}
Background: Differentiating combined pulmonary fibrosis with emphysema (CPFE) from pure emphysema can be challenging on high-resolution computed tomography (HRCT). This has antifibrotic therapy implications.

Methods: Twenty patients with suspected CPFE underwent late gadolinium-enhanced (LGE) thoracic magnetic resonance imaging (LGE-MRI) and HRCT. Data from twelve healthy control subjects from a previous study who underwent thoracic LGE-MRI were included for comparison. Quantitative LGE signal intensity (SI) was retrospectively compared in regions of fibrosis and emphysema in CPFE patients to similar lung regions in controls. Qualitative comparisons for the presence/extent of reticulation, honeycombing, and traction bronchiectasis between LGE-MRI and HRCT were assessed by two readers in consensus.

Results: There were significant quantitative differences in fibrosis SI compared to emphysema SI in CPFE patients (25.8, IQR 18.4-31.0 versus 5.3, IQR 5.0-8.1, $p<0.001$ ). Significant differences were found between LGE-MRI and HRCT in the extent of reticulation (12.5, IQR 5.0-20.0 versus 25.0, IQR 15.0-26.3, $p=0.038$ ) and honeycombing (5.0, IQR 0.0-10.0 versus 20.0, IQR 10.6-20.0, $p=0.001$ ) but not traction bronchiectasis (10.0, IQR 5-15 versus 15.0, IQR 5-15, $p=0.878$ ). Receiver operator curve analysis of fibrosis $\mathrm{SI}$ compared to similarly located regions in control subjects showed an area under the curve of $0.82(p=0.002$ ). A SI cutoff of 19 yielded a sensitivity of $75 \%$ and specificity of $86 \%$ in differentiating fibrosis from similarly located regions in control subjects.
\end{abstract}

Conclusion: LGE-MRI can differentiate CPFE from pure emphysema and may be a useful adjunct test to HRCT in patients with suspected CPFE.

Keywords: Bronchiectasis, Magnetic resonance imaging, Pulmonary emphysema, Pulmonary fibrosis, Tomography (x-ray computed)

\footnotetext{
* Correspondence: jdodd@svhg.ie

'Department of Radiology, St. Vincent's University Hospital, Elm Park, Dublin

4, Ireland

${ }^{2}$ School of Medicine, University College Dublin, Dublin, Ireland

Full list of author information is available at the end of the article
}

\section{Springer Open}

(c) The Author(s). 2020 Open Access This article is licensed under a Creative Commons Attribution 4.0 International License, which permits use, sharing, adaptation, distribution and reproduction in any medium or format, as long as you give appropriate credit to the original author(s) and the source, provide a link to the Creative Commons licence, and indicate if changes were made. The images or other third party material in this article are included in the article's Creative Commons licence, unless indicated otherwise in a credit line to the material. If material is not included in the article's Creative Commons licence and your intended use is not permitted by statutory regulation or exceeds the permitted use, you will need to obtain permission directly from the copyright holder. To view a copy of this licence, visit http://creativecommons.org/licenses/by/4.0/. 


\section{Key points}

- Late gadolinium-enhanced magnetic resonance imaging (LGE-MRI) can differentiate combined pulmonary fibrosis and emphysema from pure emphysema.

- LGE-MRI is not as accurate as high-resolution computed tomography (HRCT) in characterising reticulation or honeycombing.

- LGE-MRI may be a useful adjunct test to HRCT in differentiating combined pulmonary fibrosis and emphysema from pure emphysema.

\section{Background}

Patients with combined pulmonary fibrosis and emphysema (CPFE) are characterised by a combination of fibrosis typically in the lower lobes and emphysema typically in the upper lobes $[1,2]$. Identifying fibrosis in emphysema patients carries important prognostic and treatment implications and has been associated with an increased risk of pulmonary hypertension, progressive emphysema [3], and a higher all-cause mortality than either emphysema or fibrosis alone [4]. In the era of antifibrotic therapy, it has become even more relevant to differentiate those patients with combined fibrosis and emphysema from those with pure emphysema [5-7].

High-resolution CT (HRCT) is the most widely used imaging modality to diagnose CPFE [8]. A combination of reticulation and honeycombing are the principal fibrotic findings on HRCT. However, accurately identifying regions of definite fibrosis on HRCT can sometimes be challenging. Mimickers of honeycombing such as paraseptal emphysema may cause difficulty in confidently and accurately diagnosing cystic lung disease [9]. This is also true of cases with alternative fibrotic patterns to usual interstitial pneumonia [10]. An added issue is that spirometry may be difficult to interpret in CPFE, often showing appearances mimicking improvement in airflow obstruction because of progressive decreases in forced vital capacity over time [11]. Other patients may present with clinically suspected interstitial pulmonary fibrosis (IPF) but with obstructive spirometry. In both instances, accurate imaging of the underlying disease processes is important for diagnosis and treatment.

Cardiac MRI has become the reference-standard imaging test for detecting myocardial fibrosis [12]. A specific late gadolinium-enhanced (LGE) electrocardiographically gated segmented inversion recovery sequence allows the accurate depiction of fibrosis within the myocardium [13, 14]. The sequence relies on the concentration of the gadolinium-based contrast agent within the enlarged extracellular space of fibrotic tissue relative to normal myocardium.
It has been previously shown that pulmonary fibrosis can also be detected using this LGE-MRI in patients with IPF [15]. A key feature of this method, similar to myocardial fibrosis, is that it is the histopathological characteristics of fibrotic tissue, rather than its anatomical features, that allow the depiction of the fibrosis. In this study, we hypothesised that in patients with suspected CPFE, LGE-MRI would demonstrate contrast enhancement in regions of pulmonary fibrosis and no enhancement in regions of emphysema, allowing the differentiation of fibrosis from emphysema.

\section{Methods}

In this study, we retrospectively took into consideration the LGE-MRI scans acquired between April 2016 and March 2019 in 20 patients with CPFE suspected at HRCT. Patients were selected if there was difficulty in differentiating fibrosis from emphysema on HRCT in a multidisciplinary conference setting. This typically arose where lower lobe cysts were subpleural in location, had perceptible walls, and were round and clustered, features that are frequently seen in both fibrosis and emphysema [9]. Exclusion criteria included an acute chest infection or acute exacerbation of lung disease at the time of the investigations, contraindications to gadolinium-based contrast agents such as renal failure, and contraindications to MRI such as severe claustrophobia. The hospital Ethics Board approved the study and written informed consent was waived for this retrospective study. Data from twelve healthy control subjects from a previous study [15] who underwent LGE-MRI were included in the analysis for comparison. Body mass index was recorded for all subjects. Spirometry results were recorded when available. A flow chart diagram is provided in Fig. 1.

\section{High-resolution CT protocol}

HRCT scans were acquired on a Siemens Sensation 64slice CT (Siemens, Erlangen, Germany) from the apices to the costophrenic angles at full inspiration at $120 \mathrm{kVp}$ and $130 \mathrm{mAs}$. All patients were scanned in the supine position. Contiguous slices were reconstructed at $1-\mathrm{mm}$ slice thickness with a $0.5-\mathrm{mm}$ increment and a $512 \times$ 512 matrix. Automatic tube current modulation was utilised for all patients. Images were reconstructed using lung windows (window width 1,500, centre -700). Images were transferred to a workstation and analysed using quantitative lung analysis software (Pulmo3D, Syngo Via, Siemens, Erlangen, Germany) for calculation of total lung capacity, mean lung density, and the total low attenuation value \% (LAV\%) of the lungs. For the LAV\% an established threshold of $-950 \mathrm{HU}$ was used to indicate emphysema [16]. 


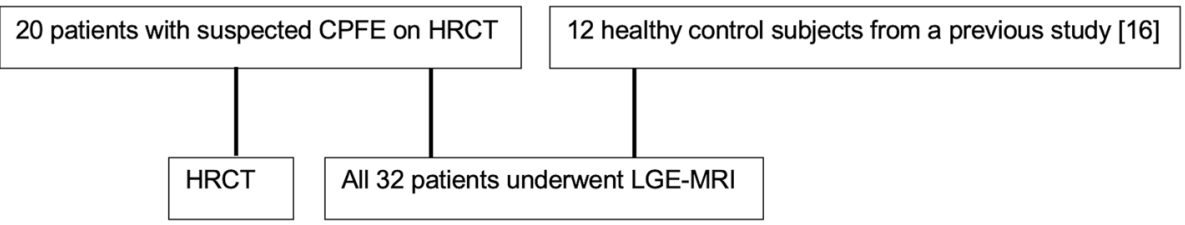

Fig. 1 Flowchart of patients and controls. CPFE, Combined pulmonary fibrosis emphysema; HRCT, High-resolution computed tomography; LGEMRI, Late gadolinium-enhanced magnetic resonance imaging

\section{Thoracic MRI protocol}

MRI scans were acquired on a 1.5 - $\mathrm{T}$ magnet (Signa 1.5 T HDX, General Electric Healthcare, Milwaukee, Wis, USA) with an eight-element phased-array cardiac coil (General Electric Healthcare, Aurora, Ohio, USA). After a localising set of axial two-dimensional steady-statefree-precession images were acquired, a bolus injection of $0.2 \mathrm{mmol} / \mathrm{kg}$ of gadoterate meglumine (Dotarem, GdDOTA, Guerbet, Paris, France) was given, followed by a saline chaser bolus of $20 \mathrm{~mL}$. This was followed $10 \mathrm{~min}$ later by a set of axial three-dimensional electrocardiographically gated segmented inversion-recovery prepared fast gradient-echo pulse sequences [17]. The 10-min delay was adapted from previously well-validated cardiac MRI protocols assessing myocardial fibrosis [18, 19]. Sequence parameters were as follows: matrix, $224 \times 128$; sensitivity encoding factor, 2; inversion time, $130-260 \mathrm{~ms}$ (individually optimised to null pulmonary artery blood signal); flip angle, $10^{\circ}$; in-plane resolution, $1.5 \times 1.5 \mathrm{~mm}^{2}$; no slice gap; section thickness $1.5 \mathrm{~mm}$ resulting in a craniocaudal volume covering $2.5 \mathrm{~cm}$ per acquisition. Median number of acquisitions to cover the lungs from apex to base was 7 (interquartile range [IQR] 6-11, depending on the height of the patient). Each acquisition required a 1015-s breath-hold. In order to null the contrast signal within the pulmonary circulation, inversion times were chosen based on the inversion time to null the blood pool in the pulmonary artery. We assessed the pulmonary artery null-time and adjusted accordingly for each acquisition, adapting similar principles from previously validated cardiac MRI approaches [20]. For patients unable to adequately breath-hold, we used nasal prongs oxygen delivery and a reduction in phase encoding steps to reduce sequence acquisition time. Scan time was recorded from the start to the end of image acquisition. Any complications such as contrast allergy were recorded.

\section{Image interpretation}

Two radiologists with 15 and 2 years of experience read all scans in consensus and in random order, blinded to all clinical data. The LGE-MRI images were read blinded to the HRCT images and vice versa. For LGE-MRI, the signal intensity (SI) of fibrosis was measured by placing regions of interest (ROI) in regions of contrast-enhancement in
CPFE patients. The ROIs were sized to avoid adjacent non-contrast lung and pulmonary vascularity. A similar process was performed for the HRCT scans. The SI of similar regions of lung in control subjects was also recorded for comparison. The SI of regions of emphysema was measured by placing ROIs in the right and left upper lobes in CPFE patients. A similar process was performed for the HRCT scans. The SI of similar regions of lung in control subjects was also recorded for comparison. LGE was defined using a SI threshold of $>3$ standard deviations above the median SI of the upper lobes of the control subjects. The standard deviation of image noise was measured in an ROI outside the body to assess the contrast to noise ratio. The contrast-to-noise ratio was calculated by (median SI of the lung region of elevated SI - median SI of the upper lobe lung region)/1.5 $\times$ (standard deviation of noise), as used by others [18]. The percentage SI within pulmonary fibrosis relative to emphysema was calculated as $100 \times$ (median SI of high SI lung region - median SI of upper lobe lung region)/(median SI of upper lobe lung region, adapted from previous myocardial fibrosis techniques [18]. The scoring system by Salisbury et al. [21] was adapted to score features of pulmonary fibrosis. Briefly, readers semiquantitatively scored the presence and extent of (i) reticulation, (ii) honeycombing, and (iii) traction bronchiectasis as follows: $0(0 \%$ involvement of the lung with the feature), 1 (1-10\% involvement), 2 (1120\%), 3 (21-30\%), 4 (31-40\%), 5 (41-50\%); 6 (51-60\%), 7 (61-70\%), 8 (71-80\%), 9 (81-90\%), and 10 (91-100\%). The midpoint of each category was arbitrarily used to convert the semiquantitative (0-10) to quantitative (0-100\%) scores. For each scan, the percent involvement of each category for the right and left lungs were averaged to obtain a total lung score, and the radiologists' total lung scores were averaged to obtain total volume occupied by each feature (HRCT was the reference standard in this study). The Fleischner society nomenclature recommendations were used to define abnormalities [22]. Scan quality for CT and MRI was scored as excellent, good, poor, or uninterpretable.

\section{Statistical analysis}

Categorical data were expressed as number and percentage and continuous data as median and IQR. Comparisons of 
nominal values were performed using the $\chi^{2}$ test, and for scale values, the independent $t$ test was used. Receiver operator characteristic (ROC) analysis was used to analyse the SI difference between regions of pulmonary fibrosis in CPFE patients with similar regions in control subjects to differentiate regions of pulmonary fibrosis from regions of dependent atelectasis (a $p$ value was generated comparing the calculated AUC in the study to a hypothetical chance level AUC [= 0.5]). Sensitivity, specificity, positive and negative predictive values of LGE-MRI in characterising reticulation, honeycombing, traction bronchiectasis, and any fibrosis were calculated using HRCT as the reference standard.

All analyses were carried out using SPSS statistical software (version 13, SPSS Inc., Chicago, IL). A $p$ value $<0.05$ was considered to indicate a statistically significant result.

\section{Results}

\section{Baseline data}

The median age of the control group was significantly lower than the CPFE group (51.5 years, IQR 41.8-62.8 versus 72.0 years, IQR 67.8-77.3, $p<0.001$ ) (Table 1 ). Median body mass index showed no significant difference between patients and controls $\left(25.4 \mathrm{~kg} / \mathrm{m}^{2}, \mathrm{IQR}\right.$ $22.4-31.3$ versus $25.4 \mathrm{~kg} / \mathrm{m}^{2}$, IQR $23.0-29.2, p=0.635$ ). Spirometry was available in 14 patients and showed a median forced expiratory volume in one second of $2.4 \mathrm{~L} /$ s (IQR 2.1-3.0) and forced vital capacity of $3.1 \mathrm{~L}$ (IQR 2.5-3.8), indicating a group of patients with moderate emphysema. This was corroborated on quantitative CT with a median total lung capacity of 5.0 L (IQR 4.2-5.5), median lung density of -791 (IQR - 825-757), and median LAV\% of 5.3\% (IQR 1.4-12.3).

Table 1 Clinical characteristics of the study population

\begin{tabular}{lll}
\hline Demographics & Controls & CPFE \\
\hline Age (years) & $51.5(41.8-62.8)$ & $72.0(67.8-77.3)^{*}$ \\
Sex & 6 males & 14 males \\
BMI $\left(\mathrm{kg} / \mathrm{m}^{2}\right)$ & $25.4(23.0-29.2)$ & $25.4(22.4-31.3)$ \\
FEV 1 (L/s) & NA & $2.4(2.1-3.0)$ \\
FVC (L) & NA & $3.1(2.5-3.8)$ \\
TLC (L) & NA & $5.0(4.2-5.5)^{* *}$ \\
MLD (HU) & NA & $-791(-825-757)^{* *}$ \\
LAV (\%) & NA & $5.3(1.4-12.3)^{* *}$
\end{tabular}

Results presented as median (interquartile interval) $B M I$ Body mass index, CPFE Combined pulmonary fibrosis and emphysema, FEV 1 Forced expiratory volume in one second; FVC Forced vital capacity, LAV\% Low attenuation value percent; MLD Mean lung density, NA Not assessed, TLC Total lung capacity

${ }^{*} p<0.001$

**Derived from quantitative computed tomography

\section{Scan data}

All subjects successfully completed all MRI scans. There were no complications. Median time between LGE-MRI and HRCT was 58 days (IQR 21.8-140.0). There was a significant difference in scan time between controls and patients (26.0, IQR 23.3-30.0 versus 29.5, IQR 26.8$35.3, p=0.041)$ related to hyperinflated lungs in the CPFE patients requiring increased scan time. MRI image quality was scored as excellent in 14 , good in 13 , fair in 4 , and poor in 1 subject. CT image quality was scored as excellent in all cases.

\section{Control subjects}

In control subjects, no significant differences were found in median SI between the upper and lower lobes (7.8, IQR 7.4-8.8 versus 6.3, IQR 0-18.1, $p=0.605$ ) (Table 2). The percentage difference in SI in the lower compared to the upper lobes was $-19.2 \%$. The contrast-to-noise ratio was 2.3. Because images were blindly scored, a small number of control subjects were incorrectly scored as having pulmonary fibrosis, because of LGE in regions of dependent atelectasis. However, scores were very limited in extent, with median reticulation, honeycombing, and traction bronchiectasis extent scores of $0.5 \%$ (IQR $0.0-1.0$ ), $0.0 \%$ (IQR $0.0-0.0$ ), and $0.0 \%$ (IQR $0-1$ ), respectively.

\section{CPFE patients}

All patients with CPFE were subjectively correctly identified on LGE-MRI. Figures 2 and 3 provide imaging examples in two patients with CPFE of LGE in regions of pulmonary fibrosis in the lower lobes and an absence of LGE in regions of emphysema in the upper lobes. The contrast-to-noise ratio was 30.1. Table 2 shows the median SI in regions of fibrosis and emphysema. Qualitatively, emphysema showed no evidence of LGE-MRI enhancement in any patient. Quantitatively, significant differences were found in median SI in regions of fibrosis compared to regions of emphysema (25.8, IQR 18.431.0 versus 5.3 , IQR $5.0-8.1, p<0.001)$. ROC analysis of $\mathrm{SI}$ in fibrotic areas in the lower lobes compared to emphysematous areas in the upper lobes in CPFE patients showed an area under the curve of $0.95, p<0.0001$. Using a SI value of $\geq 12$ resulted in a sensitivity of $95 \%$ and specificity of $100 \%$ in differentiating fibrosis from

Table 2 Comparison of signal intensity between emphysema and fibrosis in CPFE patients and equivalently located regions in controls

\begin{tabular}{llrl}
\hline & Controls & \multicolumn{1}{c}{ CPFE } & $\boldsymbol{p}$ value \\
\hline Emphysema & $7.8(7.4-8.8)$ & $5.3(5.0-8.1)$ & 0.034 \\
Fibrosis & $6.3(0.0-18.1)$ & $25.8(18.4-31.0)$ & 0.001 \\
\hline
\end{tabular}

Results presented as median (interquartile range)

CPFE Combined pulmonary fibrosis and emphysema 


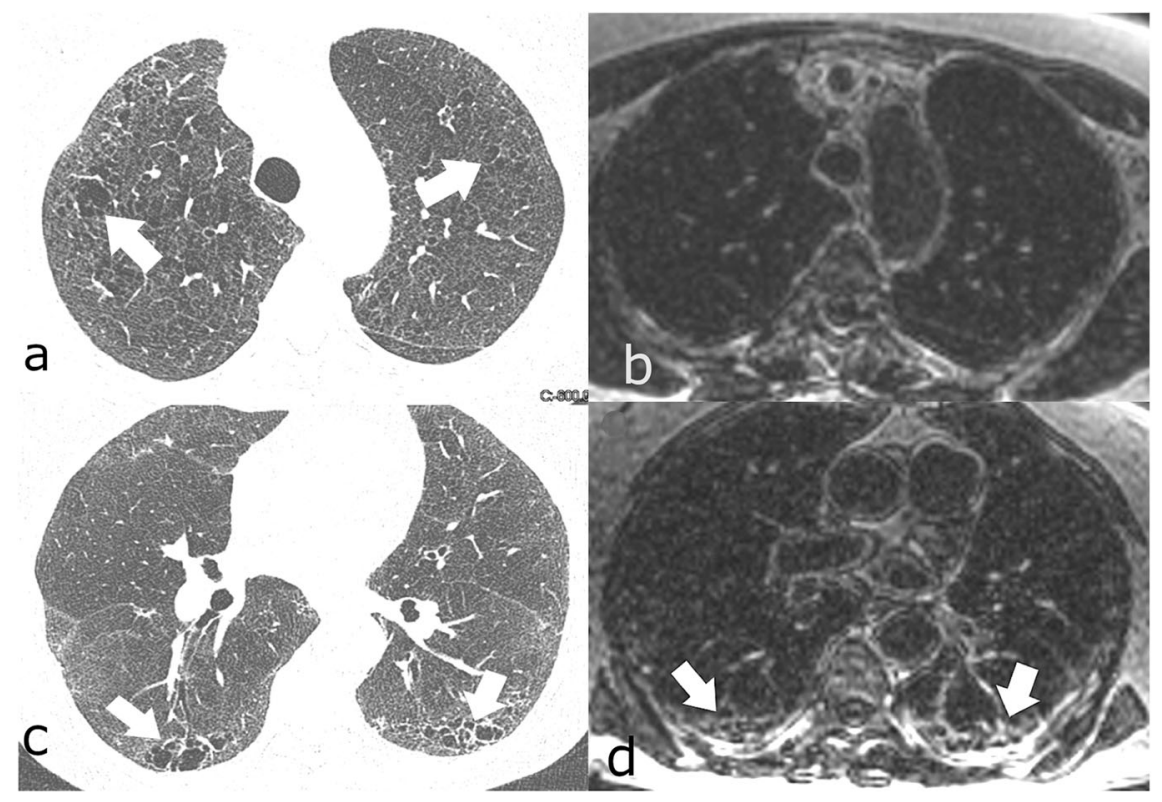

Fig. 2 Seventy-two-year-old woman with progressive breathlessness, FEV 12.4 L/s, LAV 1.5\%. a Axial high-resolution computed tomography showed mild centrilobular emphysema in the upper lobes (arrows). $\mathbf{b}$ It was unclear at multidisciplinary conference whether the abnormal regions in the lower lobes (arrows) represented emphysema or fibrosis. c Late gadolinium-enhanced magnetic resonance imaging of the upper lobes showed no contrast enhancement, but in $\mathbf{d}$, definite contrast enhancement was observed in the abnormal regions in the lower lobes (arrows) and was consistent with fibrosis. FEV ${ }_{1}$, Forced expiratory volume in one second; LAV\%, Low attenuation value percent

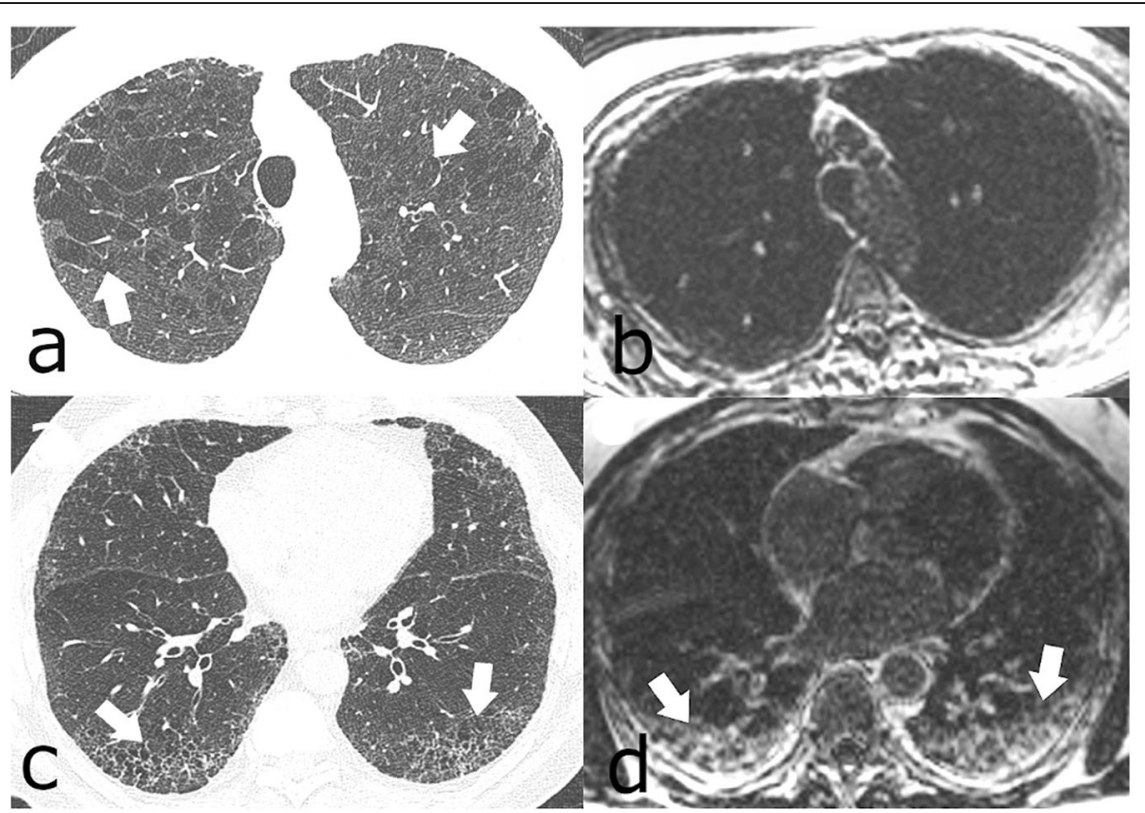

Fig. 3 Fifty-seven-year-old man with progressive breathlessness, FEV 1 2.4 L/s, LAV 7.6\%. a Axial high-resolution computed tomography showed moderate centrilobular emphysema in the upper lobes (arrows). $\mathbf{b}$ It was unclear at multidisciplinary conference whether the abnormal regions in the lower lobes (arrows) represented emphysema or fibrosis. c Late gadolinium-enhanced magnetic resonance imaging of the upper lobes showed no contrast enhancement, but in $\mathbf{d}$, definite contrast enhancement in the abnormal regions in the lower lobes (arrows) was consistent with fibrosis. FEV Forced expiratory volume in one second; LAV\%, Low attenuation value percent 
emphysema in CPFE patients (Fig. 4). The percentage of SI increase in pulmonary fibrosis compared to emphysema was $278.5 \%$ while the percentage of density increase in pulmonary fibrosis compared to emphysema on HRCT was $36.4 \%$. Significant differences were seen between LGE-MRI and HRCT in the extent of reticulation (12.5, IQR 5.0-20.0 versus 15.0-26.3, IQR 5-40, $p=$ 0.038 ) and honeycombing (5.0, IQR $0.0-10.0$ versus 20.0 , IQR 10.6-20.0, $p=0.001$ ) but not in traction bronchiectasis (10.0, IQR 5-15 versus 15.0, IQR 5.0-15, $p=0.878$ (Table 3).

\section{Comparison between groups}

Significant differences were found in $\mathrm{Si}$ in regions of fibrosis in CPFE patients compared to equivalent lower lobe regions in control subjects (25.8, IQR 18.4-31.0 versus 6.3, IQR $0.0-18.1, p=0.001$ ). There was a significantly lower SI in regions of emphysema in CPFE patients compared to equivalent upper lobe regions in control subjects (5.3, IQR 5.0-8.1 versus 7.8, IQR 7.4-8.8, $p=0.034$ ). ROC analysis of SI in regions of fibrosis in patients suspected of CPFE compared to similarly located regions in control subjects (typically in dependent regions of the lower lobes) showed an area under the curve of $0.82, p=0.002$ (Fig. 5). Using a signal cutoff intensity value of $\geq 19$ resulted in a sensitivity of $75 \%$ and specificity of $86 \%$

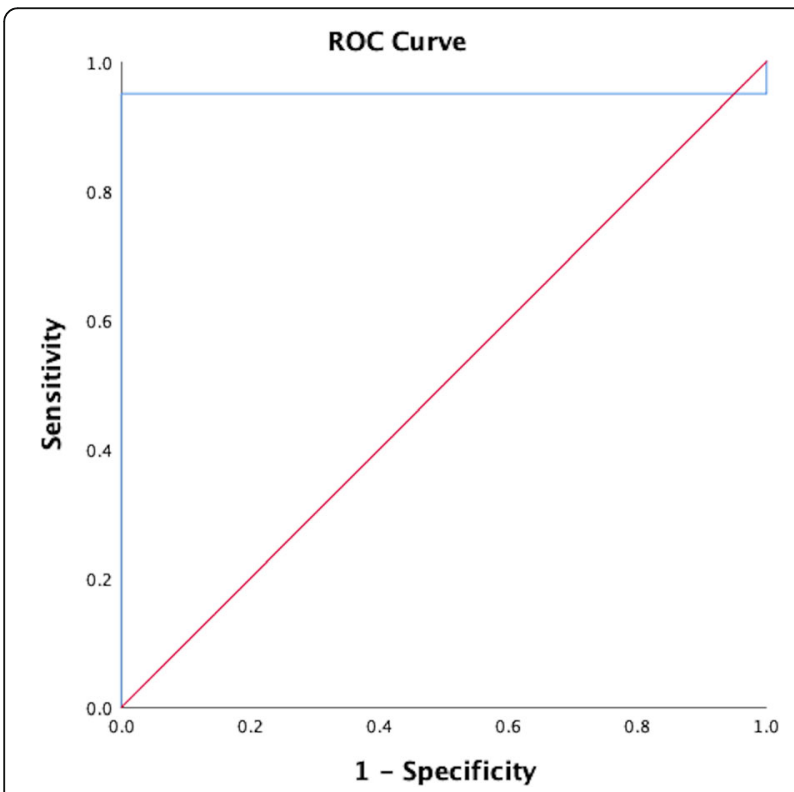

Fig. 4 Receiver operator characteristic analysis of signal intensity in regions of fibrosis in the lower lobes compared to emphysematous areas on the upper lobes in CPFE patients. The area under the curve was $0.95, p<0.001$. Using a SI value of $\geq 12$ resulted in a sensitivity of $95 \%$ and a specificity of $100 \%$ in differentiating fibrosis from emphysema in CPFE. CPFE, Combined pulmonary fibrosis and emphysema
Table 3 Comparison of extent of pulmonary abnormalities on LGE-MRI and HRCT in CPFE patients

\begin{tabular}{lrll}
\hline Abnormality & LGE-MRI & HRCT & $\boldsymbol{p}$ value \\
\hline Reticulation & $12.5(5.0-20.0)$ & $25.0(15.0-26.3)$ & 0.038 \\
Honeycombing & $5.0(0.0-10.0)$ & $20.0(10.6-20.0)$ & 0.001 \\
Traction bronchiectasis & $10.0(5.0-15.0)$ & $15.0(5.0-15.0)$ & 0.878
\end{tabular}

Results expressed as median (interquartile range). Extent of abnormalities is expressed as a percentage of total lung

CPFE Combined pulmonary fibrosis emphysema, HRCT High-resolution computed tomography, LGE-MRI Late gadolinium-enhanced magnetic resonance imaging

in differentiating regions of fibrosis from similarly located regions in control subjects. A post hoc power sample size calculation for the ROC analysis showed that for an area under the curve of 0.82 for a cohort with 20 positive and 10 negative cases and an alpha error of 0.05 yielded a power of $90 \%$. Table 4 shows that in cases of LGE-MRI where SI was $\geq 19$, LGEMRI had a high sensitivity and specificity in determining reticulation, traction bronchiectasis, and any fibrosis but a lower sensitivity (65\%) in determining honeycombing.

\section{Discussion}

The main finding from our study is that LGE-MRI may allow the differentiation of CPFE from pure emphysema alone. Its high sensitivity and specificity supports the concept that in patients where differentiating fibrosis from emphysema is challenging on HRCT, LGE-MRI may be a useful adjunct test. Although HRCT is the accepted reference standard for imaging interstitial lung fibrosis, its limitations in differentiating cystic lung disease are well published [9]. In our experience, differentiating pulmonary fibrosis from emphysema can be particularly challenging when there is an extensive paraseptal emphysema component. With several trials demonstrating the benefits of antifibrotic therapy in both IPF and non-IPF cohorts [6,23], identifying patients with combined fibrosis and emphysema from pure emphysema is becoming increasingly relevant.

As shown in a previous work [24], the most critical aspect of the MRI sequence is identifying the correct inversion time to null the pulmonary artery blood signal. Nulling the signal from the pulmonary artery blood pool allows contrast detection in regions of fibrosis but minimises it in the pulmonary circulation [15]. This is why our scan times varied considerably between patients, because it takes time to identify the correct inversion time to null the blood signal from the pulmonary artery.

There are several differences when applying LGE-MRI to the lungs compared to the heart. Firstly, in cardiac imaging, LGE-MRI relies on nulling healthy non-fibrotic myocardial tissue [25]. Fibrotic myocardium becomes detectable because the null time for normal myocardium 


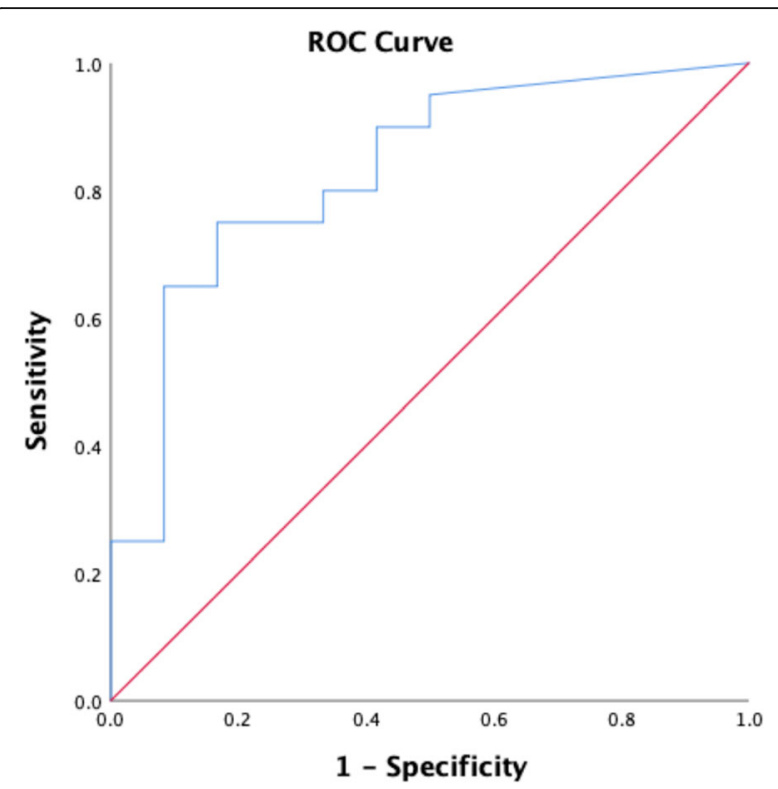

Fig. 5 Receiver operator curve of signal intensity in regions of fibrosis in CPFE patients compared to similar regions of the lungs in control subjects. The area under the curve was $0.82, p=0.002$. Using a SI value of $\geq 19$ resulted in a sensitivity of $75 \%$ and a specificity of 86\% in differentiating fibrosis in CPFE patients from dependent atelectasis in controls. CPFE, Combined pulmonary fibrosis and emphysema

differs by about $200 \mathrm{~ms}$ from the null time of myocardial fibrosis [26]. In contrast, pulmonary fibrosis has an inversion time closer to the pulmonary blood pool, the difference being $40-60 \mathrm{~ms}$. Thus, the increased SI in pulmonary fibrosis does not tend to be as high as it is in myocardial fibrosis. Nevertheless, there were significant qualitative and quantitative differences in the median percentage elevation in LGE-MRI within pulmonary fibrosis when compared to the SI of emphysema or control subjects. A much lower percentage increase in tissue density was obtained when similar measurements were repeated on HRCT.

To our knowledge, the application of LGE-MRI in patients suspected of CPFE has not been previously assessed. In a study of patients with IPF, LGE-MRI

Table 4 Sensitivity, specificity, and predictive values of LGE-MRI in determining the presence of pulmonary abnormalities on HRCT in CPFE patients

\begin{tabular}{lllll}
\hline Abnormality & Sensitivity & Specificity & PPV & NPV \\
\hline Reticulation & 0.96 & 0.82 & 0.93 & 0.90 \\
Honeycombing & 0.65 & 0.92 & 0.93 & 0.63 \\
Traction bronchiectasis & 1.00 & 0.92 & 0.95 & 1.00 \\
Any fibrosis & 1.00 & 0.85 & 0.91 & 1.00 \\
\hline
\end{tabular}

CPFE Combined pulmonary fibrosis emphysema, HRCT High-resolution computed tomography, NPV Negative predictive value, PPV Positive predictive value correctly identified all 20 IPF patients compared to controls [15]. In that study, median SI of the LGE within pulmonary fibrosis was $31.8 \pm 10.6$ versus $10.5 \pm 1.6$ for normal lung regions $(p<0.001)$, resulting in a percent SI elevation from pulmonary fibrosis of $204.8 \% \pm 90.6$. The results from the present study are similar although some controls were incorrectly scored as positive for fibrosis, related to regions of dependant atelectasis. We were unable to image patients in prone position to minimise this issue because of the requirement for vector electrocardiographic gating leads to be placed in specific anterior chest locations; placing the patient prone interfered with the electrocardiographic signal. Nevertheless, we found at ROC analysis that a SI cutoff of $\geq 19$ allowed the differentiation of CPFE from areas of dependent atelectasis in controls with high sensitivity and specificity. We also found LGE-MRI was not as accurate as HRCT at characterising reticulation and honeycombing. As shown in Table 4, LGE-MRI had a lower specificity in differentiating reticulation from honeycombing. Despite the excellent spatial resolution of the three-dimensional LGE-MRI sequence, thin septal lines $<1 \mathrm{~mm}$ in thickness were at times difficult to identify, and we were unable to differentiate thin reticulation from honeycombing in some lung regions.

Several limitations of our study are noted. We do not have tissue pathological confirmation of lung fibrosis, as we followed current recommendations from the American Thoracic Society/European Respiratory Society guidelines for patients with CPFE [2, 27]. This is an issue because some lung regions that showed contrast enhancement on LGE-MRI may have represented true fibrosis, despite not being scored as fibrosis on the corresponding HRCT images. Nevertheless, we used HRCT because it is the current consensus imaging reference standard for pulmonary fibrosis [28].

It was not our intention to assess the optimum delay between contrast injection and MRI acquisition. We applied a 10-min delay because that is the standard protocol for depicting LGE-MRI in protocols for myocardial fibrosis [19]. For pulmonary fibrosis, it may be better to wait a longer time before acquisition, which might allow further washout of contrast from the pulmonary blood pool and help depict fibrosis more optimally. Further studies are needed to optimise these timing aspects. The LGE-MRI technique does require the administration of intravenous contrast, so is not suitable in all patients, for example those with severe renal failure. We did not assess inter-reader variability or reader experience in this study, which is an important aspect to evaluate in the future. We did not assess the technique across multiple vendors and this is another aspect to assess in future studies. Finally, while we have shown that LGE-MRI allows the quantitative analysis of pulmonary fibrosis and 
emphysema, imaging biomarkers for HRCT are undergoing rapid development [29]. Future studies comparing both modalities should provide further insights into which give more information in the quantitative analysis of CPFE.

In conclusion, LGE-MRI may differentiate CPFE from pure emphysema. It may be a useful adjunct test to HRCT in patients with suspected CPFE.

\section{Abbreviations}

CPFE: Combined pulmonary fibrosis and emphysema; HRCT: High-resolution computed tomography; IPF: Interstitial pulmonary fibrosis; IQR: Interquartile range; LAV: Lung attenuation volume; LGE: Late gadolinium-enhanced; ROC: Receiver operating characteristic; ROI: Region of interest; SI: Signal intensity

\section{Authors' contributions}

The contribution of the included authors are as follows: HF, conceptualisation, investigation, writing, formal analysis, response to editor/ reviewer comments, revision; SC, investigation, formal analysis; $\mathrm{AH}$, investigation, formal analysis; RMD, investigation, writing, review, and editing; $\mathrm{GH}$, conception of the work; LL, conceptualisation of the work, methodology; SA, conceptualisation of the work; DJM, conceptualisation of the work, methodology, writing, editing; AF, conceptualisation, methodology; EMcK, conceptualisation of the work, editing; CMcC, conceptualisation, methodology; $\mathrm{MB}$, conceptualisation, writing, editing; PD, conceptualisation; $\mathrm{DL}$, conceptualisation; MK, conceptualisation, funding acquisition; JD, principal investigator; conceptualisation, methodology, supervision, funding acquisition, formal analysis; response to editor/reviewer comments; revisions. All authors have read and approved the final manuscript.

All authors have agreed both to be personally accountable for the author's own contributions and to ensure that questions related to the accuracy or integrity of any part of the work, even ones in which the author was not personally involved, are appropriately investigated, resolved, and the resolution documented in the literature.

\section{Funding}

Funding was provided by the St. Vincent's University Hospital Foundation.

\section{Availability of data and materials}

The datasets used and/or analysed during this study are available from the corresponding author on reasonable request.

\section{Ethics approval and consent to participate}

The project was approved by the Ethics Board of St. Vincent's University Hospital. Written informed consent was waived for this retrospective study Note however that data from 12 healthy control subjects from a previous study (16) were included in the analysis for comparison. In that study, ethics approval was received and written informed consent was required and obtained from all subjects.

\section{Consent for publication}

The project was approved by the Ethics Board of St. Vincent's University Hospital. Written informed consent was waived for this retrospective study. Images are entirely unidentifiable and there are no details on individuals reported within the manuscript.

\section{Competing interests}

The authors declare that they have no competing interests.

\section{Author details}

'Department of Radiology, St. Vincent's University Hospital, Elm Park, Dublin 4, Ireland. ${ }^{2}$ School of Medicine, University College Dublin, Dublin, Ireland. ${ }^{3}$ Department of Radiology, UT Southwestern Hospital, Dallas, TX, USA. ${ }^{4}$ Department of Pathology, St. Vincent's University Hospital, Dublin, Ireland. ${ }^{5}$ Department of Respiratory Medicine, St. Vincent's University Hospital, Dublin, Ireland. ${ }^{6}$ UCD Clinical Research Center, University College Dublin, Dublin, Ireland. ${ }^{7}$ Department of Radiology, National Jewish Medical and Research Center, Denver, CO, USA.
Received: 29 July 2020 Accepted: 1 October 2020

Published online: 03 November 2020

\section{References}

1. Cottin V, Nunes H, Brillet PY et al (2005) Combined pulmonary fibrosis and emphysema: a distinct underrecognised entity. Eur Respir J 26:586-593. https://doi.org/10.1183/09031936.05.00021005

2. Travis WD, Costabel U, Hansell DM et al (2013) An official American Thoracic Society/European Respiratory Society statement: update of the international multidisciplinary classification of the idiopathic interstitial pneumonias. Am J Respir Crit Care Med 188:733-748. https://doi.org/10.1164/rccm.2013081483ST

3. Matsuoka S, Yamashiro T, Matsushita S et al (2015) Morphological disease progression of combined pulmonary fibrosis and emphysema: comparison with emphysema alone and pulmonary fibrosis alone. J Comput Assist Tomogr 39:153-159. https://doi.org/10.1097/rct.0000000000000184

4. Zhang L, Zhang C, Dong F et al (2016) Combined pulmonary fibrosis and emphysema: a retrospective analysis of clinical characteristics, treatment and prognosis. BMC Pulm Med 16:137. https://doi.org/10.1186/s12890-0160300-7

5. Oltmanns U, Kahn N, Palmowski K et al (2014) Pirfenidone in idiopathic pulmonary fibrosis: real-life experience from a German tertiary referral center for interstitial lung diseases. Respiration 88:199-207. https://doi.org/ 10.1159/000363064

6. Richeldi L, du Bois RM, Raghu G et al (2014) Efficacy and safety of nintedanib in idiopathic pulmonary fibrosis. N Engl J Med 370:2071-2082. https://doi.org/10.1056/NEJMoa1402584

7. Richeldi L, Kolb M, Jouneau S et al (2020) Efficacy and safety of nintedanib in patients with advanced idiopathic pulmonary fibrosis. BMC Pulm Med 20: 3. https://doi.org/10.1186/s12890-019-1030-4

8. Pepin EW, Verma N, Mehta HJ, Mohammed TL (2018) Combined pulmonary fibrosis and emphysema. Ann Am Thorac Soc 15:110-112. https://doi.org/ 10.1513/AnnalsATS.201706-473CC

9. Koyama M, Johkoh T, Honda O et al (2003) Chronic cystic lung disease: diagnostic accuracy of high-resolution CT in 92 patients. AJR Am J Roentgenol 180:827-835. https://doi.org/10.2214/ajr.180.3.1800827

10. Alsumrain M, De Giacomi F, Nasim F et al (2019) Combined pulmonary fibrosis and emphysema as a clinicoradiologic entity: characterization of presenting lung fibrosis and implications for survival. Respir Med 146:106112. https://doi.org/10.1016/j.rmed.2018.12.003

11. Kitaguchi Y, Fujimoto K, Hayashi R, Hanaoka M, Honda T, Kubo K (2013) Annual changes in pulmonary function in combined pulmonary fibrosis and emphysema: over a 5-year follow-up. Respir Med 107:1986-1992. https://doi. org/10.1016/j.rmed.2013.06.015

12. Kim RJ, Wu E, Rafael A et al (2000) The use of contrast-enhanced magnetic resonance imaging to identify reversible myocardial dysfunction. N Engl J Med 343:1445-1453. https://doi.org/10.1056/NEJM200011163432003

13. Kim RJ, Fieno DS, Parrish TB et al (1999) Relationship of MRI delayed contrast enhancement to irreversible injury, infarct age, and contractile function. Circulation 100:1992-2002. https:/doi.org/10.1161/01.cir.100.19.1992

14. Shan K, Constantine G, Sivananthan M, Flamm SD (2004) Role of cardiac magnetic resonance imaging in the assessment of myocardial viability. Circulation 109:1328-1334. https://doi.org/10.1161/01.CIR.0000120294.67948.E3

15. Lavelle LP, Brady D, McEvoy S et al (2017) Pulmonary fibrosis: tissue characterization using late-enhanced MRI compared with unenhanced anatomic high-resolution CT. Diagn Interv Radiol 23:106-111. https://doi. org/10.5152/dir.2016.15331

16. Wang Z, Gu S, Leader JK et al (2013) Optimal threshold in CT quantification of emphysema. Eur Radiol 23:975-984. https://doi.org/10.1007/s00330-012$2683-z$

17. Goetti R, Kozerke S, Donati OF et al (2011) Acute, subacute, and chronic myocardial infarction: quantitative comparison of $2 \mathrm{D}$ and $3 \mathrm{D}$ late gadolinium enhancement MR imaging. Radiology 259:704-711. https://doi. org/10.1148/radiol.11102216

18. Simonetti OP, Kim RJ, Fieno DS et al (2001) An improved MR imaging technique for the visualization of myocardial infarction. Radiology 218:215223. https://doi.org/10.1148/radiology.218.1.r01ja50215

19. Kim RJ, Shah DJ, Judd RM (2003) How we perform delayed enhancement imaging. J Cardiovasc Magn Reson 5:505-514. https://doi.org/10.1081/jcmr120022267 
20. Mahrholdt H, Wagner A, Holly TA et al (2002) Reproducibility of chronic infarct size measurement by contrast-enhanced magnetic resonance imaging. Circulation 106:2322-2327. https://doi.org/10.1161/01.cir. $0000036368.63317 .1 \mathrm{C}$

21. Salisbury ML, Lynch DA, van Beek EJ et al (2017) Idiopathic pulmonary fibrosis: the association between the adaptive multiple features method and fibrosis outcomes. Am J Respir Crit Care Med 195:921-929. https://doi. org/10.1164/rccm.201607-13850C

22. Hansell DM, Bankier AA, MacMahon H, McLoud TC, Muller NL, Remy J (2008) Fleischner Society: glossary of terms for thoracic imaging. Radiology 246: 697-722. https://doi.org/10.1148/radiol.2462070712

23. Flaherty KR, Wells AU, Cottin V et al (2019) Nintedanib in progressive fibrosing interstitial lung diseases. N Engl J Med 381:1718-1727. https://doi. org/10.1056/NEJMoa1908681

24. Thomson LE, Kim RJ, Judd RM (2004) Magnetic resonance imaging for the assessment of myocardial viability. J Magn Reson Imaging 19:771-788. https://doi.org/10.1002/jmri.20075

25. Rajiah P, Desai MY, Kwon D, Flamm SD (2013) MR imaging of myocardial infarction. Radiographics 33:1383-1412. https://doi.org/10.1148/rg. 335125722

26. Mahrholdt H, Wagner A, Judd RM, Sechtem U (2002) Assessment of myocardial viability by cardiovascular magnetic resonance imaging. Eur Heart J 23:602-619. https://doi.org/10.1053/euhj.2001.3038

27. Raghu G, Remy-Jardin M, Myers JL et al (2018) Diagnosis of idiopathic pulmonary fibrosis. an official ATS/ERS/JRS/ALAT clinical practice guideline. Am J Respir Crit Care Med 198:e44-e68. https://doi.org/10.1164/rccm. 201807-1255ST

28. Lederer DJ, Martinez FJ (2018) Idiopathic pulmonary fibrosis. N Engl J Med 378:1811-1823. https://doi.org/10.1056/NEJMra1705751

29. Wu X, Kim GH, Salisbury ML et al (2019) Computed tomographic biomarkers in idiopathic pulmonary fibrosis. The future of quantitative analysis. Am J Respir Crit Care Med 199:12-21. https://doi.org/10.1164/rccm.201803-0444PP

\section{Publisher's Note}

Springer Nature remains neutral with regard to jurisdictional claims in published maps and institutional affiliations.

\section{Submit your manuscript to a SpringerOpen ${ }^{\circ}$ journal and benefit from:}

- Convenient online submission

- Rigorous peer review

- Open access: articles freely available online

- High visibility within the field

- Retaining the copyright to your article

Submit your next manuscript at $\boldsymbol{\nabla}$ springeropen.com 